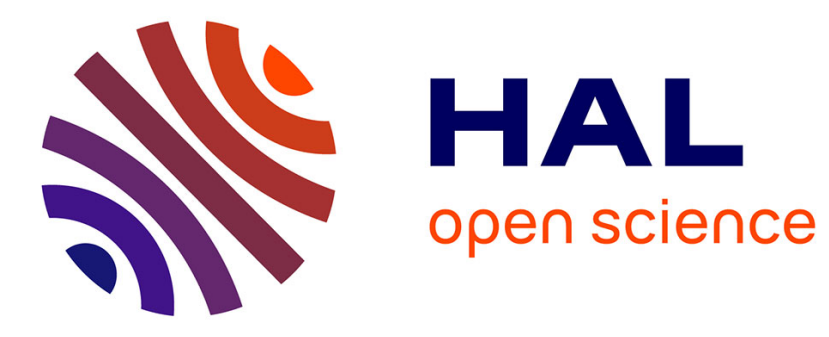

\title{
Generation of spin waves via spin-phonon interaction in a buried dielectric thin film
}

\author{
Marwan Deb, Elena Popova, Steffen Peer Zeuschner, Michel Hehn, Niels \\ Keller, Stéphane Mangin, Grégory Malinowski, Matias Bargheer
}

\section{- To cite this version:}

Marwan Deb, Elena Popova, Steffen Peer Zeuschner, Michel Hehn, Niels Keller, et al.. Generation of spin waves via spin-phonon interaction in a buried dielectric thin film. Physical Review B, 2021, 103, pp.024411. 10.1103/physrevb.103.024411 . hal-03113275

\section{HAL Id: hal-03113275 \\ https://hal.univ-lorraine.fr/hal-03113275}

Submitted on 18 Jan 2021

HAL is a multi-disciplinary open access archive for the deposit and dissemination of scientific research documents, whether they are published or not. The documents may come from teaching and research institutions in France or abroad, or from public or private research centers.
L'archive ouverte pluridisciplinaire HAL, est destinée au dépôt et à la diffusion de documents scientifiques de niveau recherche, publiés ou non, émanant des établissements d'enseignement et de recherche français ou étrangers, des laboratoires publics ou privés. 


\title{
Generation of spin waves via spin-phonon interaction in a buried dielectric thin film
}

\author{
Marwan Deb $\odot,{ }^{1, *}$ Elena Popova, ${ }^{2}$ Steffen Peer Zeuschner $\odot,{ }^{1,3}$ Michel Hehn, ${ }^{4}$ Niels Keller, ${ }^{2}$ Stéphane Mangin, ${ }^{4}$ \\ Gregory Malinowski, ${ }^{4}$ and Matias Bargheer ${ }^{1,3}$ \\ ${ }^{1}$ Institut für Physik und Astronomie, Universität Potsdam, Karl-Liebknecht-Straße 24-25, 14476 Potsdam, Germany \\ ${ }^{2}$ Groupe d'Etude de la Matière Condensée (GEMaC), CNRS UMR 8635, Université Paris-Saclay, 78035 Versailles, France \\ ${ }^{3}$ Helmholtz Zentrum Berlin für Materialien und Energie GmbH, Albert-Einstein-Straße 15, 12489 Berlin, Germany \\ ${ }^{4}$ Institut Jean Lamour (IJL), CNRS UMR 7198, Université de Lorraine, 54506 Vandœuvre-lès-Nancy, France
}

(Received 23 October 2020; revised 21 December 2020; accepted 23 December 2020; published 11 January 2021)

\begin{abstract}
In this paper, we investigate the magnetic, optical, and lattice responses of a Pt/Cu/ $\mathrm{Bi}_{1} \mathrm{Y}_{2} \mathrm{Fe}_{5} \mathrm{O}_{12} / \mathrm{Gd}_{3} \mathrm{Ga}_{5} \mathrm{O}_{12}$ heterostructure to femtosecond laser excitation of the opaque $\mathrm{Pt} / \mathrm{Cu}$ metallic bilayer. The electronic excitation generates coherent and incoherent phonons, which trigger high-frequency standing spin waves (SWs) in the dielectric $\mathrm{Bi}_{1} \mathrm{Y}_{2} \mathrm{Fe}_{5} \mathrm{O}_{12}$ layer via a phonon-induced change of magnetic anisotropy. We find that the incoherent phonons (heat) can induce a fast $(<1 \mathrm{ps})$ and slow $(>1000 \mathrm{ps})$ decrease of the magnetic order by different spin-phonon interaction scenarios. These results open perspectives for generating high-frequency SWs in buried magnetic garnets.
\end{abstract}

DOI: 10.1103/PhysRevB.103.024411

\section{INTRODUCTION}

Controlling the spin dynamics at the fastest speed and with the highest efficiency has always been one of the ultimate goals of modern magnetism, as it constitutes a fundamentally important challenge with a potential high impact on data storage and processing technologies. Since the discovery of the subpicosecond demagnetization in nickel [1], laserinduced ultrafast magnetization dynamics have attracted a lot of attention and have become the rapidly developing field of femtomagnetism [2,3]. Indeed, over the past 25 years, it was shown that femtosecond laser pulses can trigger many important magnetic phenomena such as all-optical magnetization switching [4,5], ultrafast magnetic phase transition [6-8], and the generation of collective spin oscillations $[9,10]$. These results have provided important insight about fundamental mechanisms behind the excitation of spins with light. It was demonstrated that thermal effects play an important role in the majority of ultrafast magnetic processes induced in conducting $[1,11,12]$ and semiconducting [13-16] magnets, while in magnetic insulators, the ultrafast control of spin dynamics can occur via nonthermal mechanisms like the inverse Faraday effect $[10,17,18]$, the Cotton-Mouton effect [19], and the photo-induced magnetic anisotropy [20-23].

These results have motivated researchers to explore alternative possibilities to control the magnetization using other ultrashort stimuli like picosecond strain pulses and hot-electron pulses. For instance, the propagation of an ultrashort hot-electron pulse in conducting magnets can induce an ultrafast demagnetization [24-26] and a full magnetization reversal $[25,26]$ via thermal effects. Picosecond acoustic pulses can trigger a homogeneous collective spin oscillation

*Corresponding author: madeb@uni-potsdam.de
( $k=0$, i.e., ferromagnetic resonance mode) via inverse magnetostriction in conducting [27-29], semiconducting [30], and insulating [31] magnetic materials. Very recently, the geometry for exciting spin dynamics indirectly via pumping a $\mathrm{Pt} / \mathrm{Cu}$ bilayer that protects a magnetic layer from optical excitation $[24,25]$ was investigated by ultrafast $x$-ray diffraction (UXRD) [32]. These experiments quantify the arrival of hot electrons, strain waves, and vibrational heat in a magnetic $\mathrm{Ni}$ layer.

The collective spin oscillations, known also as spin waves (SWs) or magnons, represent one of the most promising approaches toward the development of more energy-efficient and faster data transport and processing technology than the current charge-based semiconductor-based technology [33]. This is due to their very useful physical properties at room temperature, such as the low-energy dissipation, the high-frequency spectrum from $\mathrm{GHz}$ to $\mathrm{THz}$, and the tunable wavelength down to nanoscales [34]. A key requirement for high-speed applications is to generate SWs that combine low damping with high frequency. Nanoscale thin films of yttrium iron garnet $\left(\mathrm{Y}_{3} \mathrm{Fe}_{5} \mathrm{O}_{12}\right.$, YIG) with its intrinsic low magnetic damping constant [35] are particularly interesting for these applications. The frequency of exchange standing SW (SSW) modes increases quadratically with the mode number $n$ and with decreasing thickness $d$ of the nanolayer. A moderate exchange stiffness $D_{\text {ex }}$ can thus support the high frequency $\sim D_{\mathrm{ex}}(\pi n / d)^{2}$ required by applications [36,37].

Generating SSWs requires nonuniform excitation across the film thickness [38], which is very challenging to induce using conventional microwave antennae [38-40]. On the other hand, for around 15 years, the excitation of SSWs with femtosecond laser pulses has been limited to conducting $[9,41,42]$ and semiconducting $[13,15,16]$ magnets. In our recent papers, we demonstrated that a femtosecond laser pulse can excite SSWs in insulating magnetic films of bismuth-substituted 
YIG with high in-plane magnetic anisotropy $[43,44]$. In this context, an important fundamental study is to investigate the possibility to take advantage of femtosecond laser pulses to trigger high-frequency SSWs in bismuth-substituted YIG films buried below a thick metallic structure. This configuration is of utmost importance since it allows exploring the effects of picosecond strain excitation and heat transfer across a normal metal-magnetic insulator interface on the magnetic properties.

Here, we report an experimental investigation of the ultrafast magnetization dynamics in a dielectric film of $\mathrm{Bi}_{1} \mathrm{Y}_{2} \mathrm{Fe}_{5} \mathrm{O}_{12}$ (Bi-YIG) with high in-plane magnetic anisotropy and buried below a thick $\mathrm{Cu} / \mathrm{Pt}$ metallic bilayer. We demonstrate that the indirect excitation of the Bi-YIG layer via exclusive absorption of light in the metal triggers high-frequency SSWs in the insulating Bi-YIG. Two mechanisms mediate this indirect excitation of SSWs: (i) Inverse magnetostriction changes the magnetic anisotropy proportional to lattice constant changes in the form of propagating strain waves. (ii) The lattice-heating additionally changes the magnetic anisotropy via the temperature dependence of the anisotropy constants. The strain pulse (coherent phonon) launched in the metal, traversing the Bi-YIG layer within 50 ps, and the rapid propagation of heat (incoherent phonons) can both excite SSWs. We suggest that phonons created at the $\mathrm{Cu} / \mathrm{Bi}-\mathrm{YIG}$ interface via hot electrons reflected at the metalinsulator interface additionally induce a fast $(<1 \mathrm{ps})$ change of the magnetic order. The slower timescale ( $>1 \mathrm{~ns}$ ) on which the magnetic order is further reduced can be attributed to a combination of slow phonon-spin coupling and heat transport into the Bi-YIG layer. Our model is based on the experimental results from time-resolved magneto-optical Kerr effect (TRMOKE) and UXRD.

\section{SAMPLE CHARACTERIZATION AND EXPERIMENTAL METHODS}

The sample studied in this paper was based on a 135-nmthick $\mathrm{Bi}_{1} \mathrm{Y}_{2} \mathrm{Fe}_{5} \mathrm{O}_{12}$ single crystalline film grown by pulsed laser deposition on a gadolinium gallium garnet (GGG) (100) substrate [45]. To address the effect of a phononic excitation on ultrafast magnetization dynamics that excludes optical excitation, we deposited a thick $\mathrm{Pt}(5 \mathrm{~nm}) / \mathrm{Cu}(100 \mathrm{~nm})$ nonmagnetic metallic bilayer on top of the Bi-YIG film using direct current magnetron sputtering [Fig. 2(b)]. The top Pt layer was added due to its high absorption at $800 \mathrm{~nm}$ compared with $\mathrm{Cu}$, which also ensured an efficient generation of a hot-electron pulse [24,25,32]. Experimentally, the transmission of the pump light was less than $10^{-3}$. The $\mathrm{Cu}$ was chosen for its high hot-electron lifetime $[24,25,46]$. Thus, the 100$\mathrm{nm}$-thick $\mathrm{Cu}$ layer protected the Bi-YIG film from the direct light excitation, while the hot-electron pulses propagated until reaching the back side of the $\mathrm{Cu}$ layer [24,25,32].

Figure 1 shows the UXRD study of the heterostructure, performed with femtosecond x-ray diffraction using a tabletop laser-driven plasma source [47]. In full analogy to the recent publication on a $\mathrm{Pt} / \mathrm{Cu} / \mathrm{Ni}$ heterostructure [32], we were able to model the time-resolved strain in $\mathrm{Pt}, \mathrm{Cu}$, and Bi-YIG with a two-temperature model in combination with a one-dimensional masses-and-springs model, using the
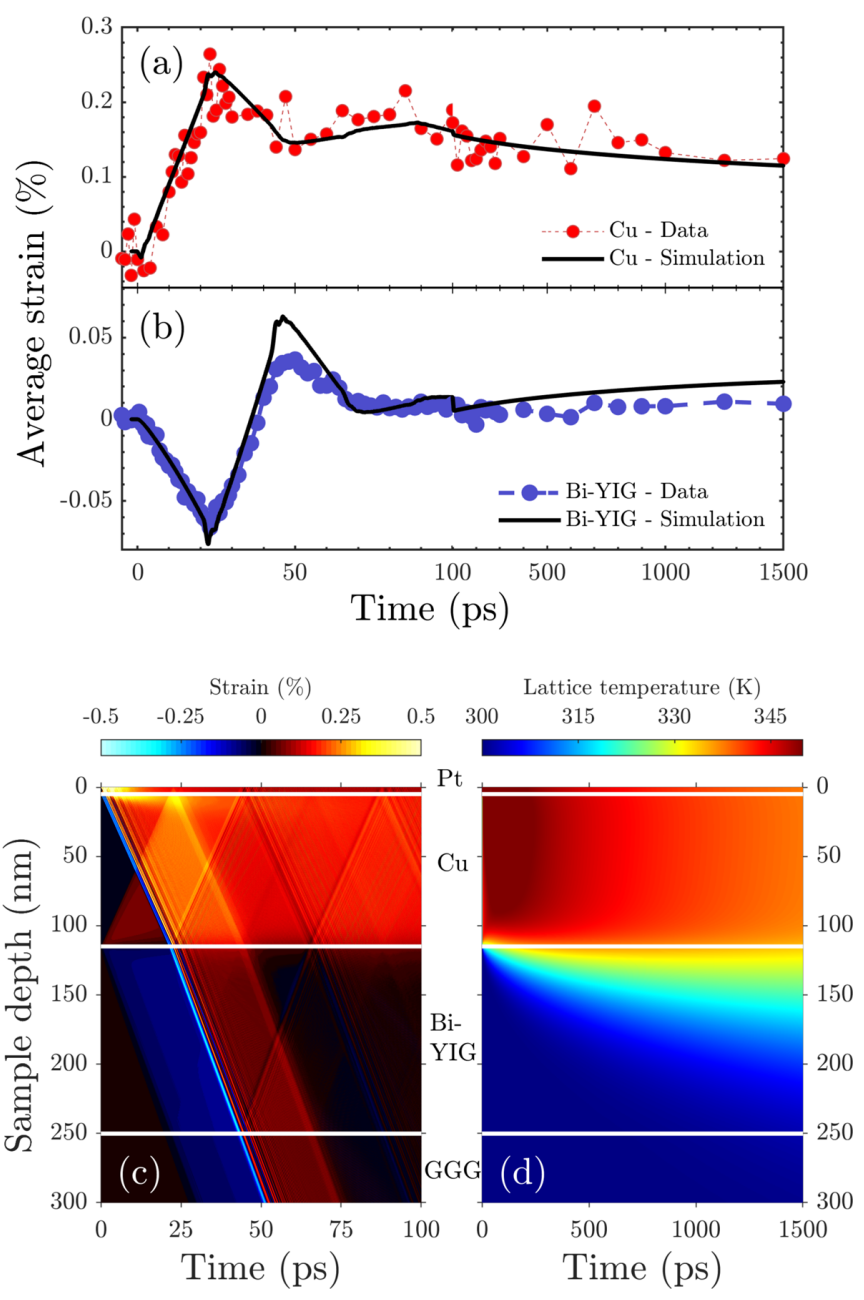

FIG. 1. Laser-induced lattice dynamics in the $\mathrm{Pt} / \mathrm{Cu} /$ $\mathrm{Bi}_{1} \mathrm{Y}_{2} \mathrm{Fe}_{5} \mathrm{O}_{12} / \mathrm{Gd}_{3} \mathrm{Ga}_{5} \mathrm{O}_{12}$ heterostructure. Average strain in (a) the $\mathrm{Cu}$ layer and (b) BI-YIG layer as measured by ultrafast X-ray diffraction (UXRD). Calculated spatiotemporal maps of (c) the strain and (d) temperature in the heterostructure. The solid lines in (a) and (b) are results of our modeling. The UXRD measurements were performed at room temperature with a pump wavelength of $800 \mathrm{~nm}$, a pulse duration of $50 \mathrm{fs}$, and an incident pump energy density of $5 \mathrm{~mJ} / \mathrm{cm}^{2}$.

software package udkm1Dsim-toolbox [48]. The twotemperature model includes an enhanced electronic heat conductivity $\kappa_{e}=\kappa_{0} \times T_{e} / T_{p h}$ compared with the equilibrium value $\kappa_{0}$. This enhancement is due to the reduction of the electron-phonon scattering channel if the lattice of $\mathrm{Cu}$ is cold compared with its electronic system [32]. The main difference to the previous paper is that the magnetic layer now is the insulating Bi-YIG, which cannot accept the thermal energy in the form of hot electrons. Instead, the hot electrons in $\mathrm{Cu}$ can excite optical phonons in Bi-YIG when they scatter at the interface, which quickly decay. In addition, heat can be transported into the Bi-YIG film via incoherent acoustic phonons from the $\mathrm{Cu}$. Figures 1(a) and 1(b) show the measured timeresolved average strains $\bar{\eta}_{\mathrm{Cu}}(t)$ of the $\mathrm{Cu}$ layer and $\bar{\eta}_{\mathrm{Bi}-\mathrm{YIG}}(t)$ of the Bi-YIG layer. As a first approximation, we disregard the direct transfer of energy from $\mathrm{Cu}$ electrons to $\mathrm{Bi}-\mathrm{YIG}$ phonons and calculate the spatiotemporal lattice strain $\eta(z, t)$ from the 
(a)

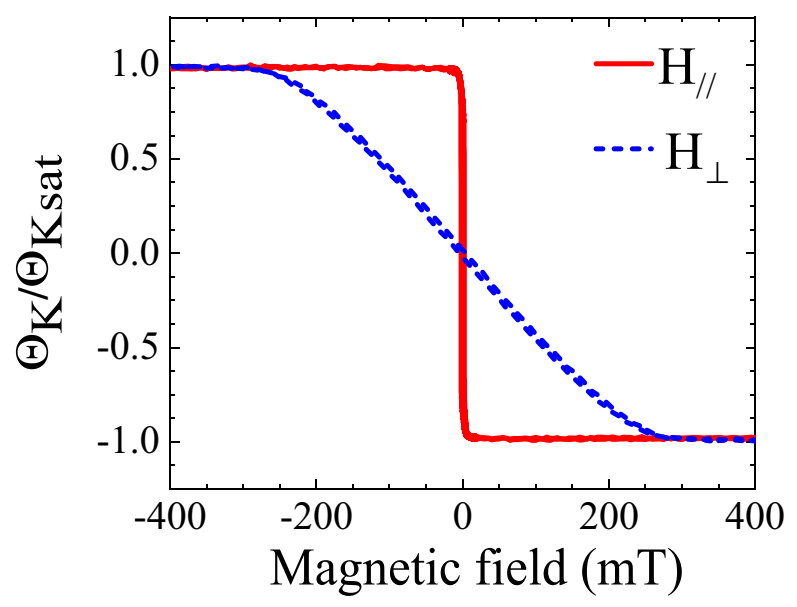

(b)

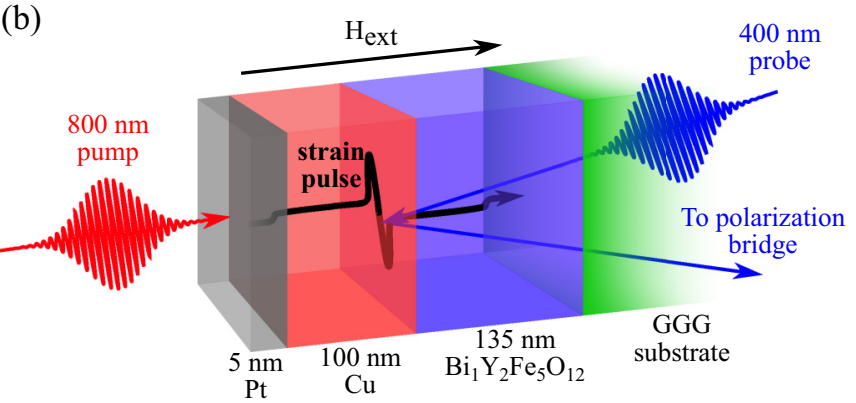

FIG. 2. Static room temperature magnetic properties of the garnet sample and the experimental configuration. (a) Normalized hysteresis loop measured in polar (dashed line) and longitudinal (solid line) configurations. (b) Sketch of the pump-probe timeresolved magneto-optical configuration that allows studying the ultrafast magnetization dynamics induced by coherent and incoherent phonons. The sketch also shows a snapshot of the bipolar strain pulse that traverses the Bi-YIG layer as modeled in Fig. 1(c). It contains a bipolar pulse with a broad, low-amplitude contribution from $\mathrm{Cu}$ excitation and a sharp high-amplitude contribution from $\mathrm{Pt}$ excitation.

temperature $T(z, t)$ in the heterostructure [see Figs. 1(c) and 1(d)]. In our simulation, we used the same parameters for Pt and $\mathrm{Cu}$ in Ref. [32] except that the $\mathrm{Cu}$ thickness was $110 \mathrm{~nm}$ to match the timings of the coherent strain signals. For Bi-YIG, we used a longitudinal sound velocity $V_{\mathrm{Bi}-\mathrm{YIG}}^{L}=6300 \mathrm{~m} / \mathrm{s}$ [49,50], a phonon heat capacity $C_{\mathrm{Bi}-\mathrm{YIG}}^{p h}=2.9 \mathrm{~J} / \mathrm{m}^{3} \mathrm{~K}[51]$, a phonon heat conductivity $\kappa_{\mathrm{Bi}-\mathrm{YIG}}^{p h}=6 \mathrm{~W} / \mathrm{mK}$ [51], and a Grüneisen constant $\Gamma_{\mathrm{Bi}-\mathrm{YIG}}^{s}=1$ [50]. The modeling of the average lattice strain is plotted as solid lines in Figs. 1(a) and 1(b), showing a reasonable agreement with the experimental data. We can use the transient temperature and strain which was calibrated to the UXRD experiment to discuss the excitation channels of the SWs. In Fig. 2(b), we display the calculated shape of the strain pulse that traverses the Bi-YIG layer, i.e., the time-dependent local strain that modulates each unit cell. It is a vertical cut through Fig. 1(c) at 25 ps. It contains a low-amplitude feature originating from the expan- sion of the Cu layer [Fig. 1(a)], which induces the immediate decrease of the strain in Fig. 1(b), starting within less than 1 ps. At this time, the heat energy transported by the hot electrons through $\mathrm{Cu}$ arrived at the Bi-YIG interface. The sharper high-amplitude feature originated from the expansion of the thin Pt transducer. Both features of the strain pulse together with the thermal expansion are shown in the strain map of Fig. 1(c), which highlights the spatiotemporal dynamics of the lattice strain in the heterostructure. The heat diffusion is illustrated by the phonon temperature profile [Fig. 1(d)]. This phonon heat energy density was the main component of the stress that drove the strain $[32,48,50]$. More importantly, Fig. 1(d) shows that the heating of the Bi-YIG layer is strongly inhomogeneous. Although the diffusion reached the back end of the Bi-YIG layer only at $\sim 2 \mathrm{~ns}$, the temperature rose by more than $40 \mathrm{~K}$ within $100 \mathrm{ps}$ in the first $10 \mathrm{~nm}$ behind the $\mathrm{Cu}$ interface [see Fig. 1(d)].

The static magnetic properties of the sample are characterized using the MOKE. The normalized polar and longitudinal Kerr hysteresis loops are shown in Fig. 2(a). The out-of-plane normalized remanence $\left(M_{r} / M_{s}\right)$ was about 0.02 , which is very small compared with the in-plane one of 0.85 . The out-ofplane saturation field $(\sim 260 \mathrm{mT})$ was about 50 times larger than the in-plane one $(\sim 5 \mathrm{mT})$. These results reveal a strong in-plane magnetic anisotropy. The origin of this in-plane easy axis of magnetization is a large growth-induced anisotropy field [52,53], which has the value of $-83 \mathrm{mT}$ in the case of our film. The TR-MOKE and reflectivity measurements were investigated with the all-optical pump-probe configuration sketched in Fig. 2(b). We have employed a femtosecond laser pulse issued from an amplified Ti-Sapphire laser system operating at a $5-\mathrm{kHz}$ repetition rate and delivering $35-\mathrm{fs}$ pulses at $800 \mathrm{~nm}$ to generate the pump and the probe beams. The pump beam was kept at the fundamental wavelength of the amplifier at $800 \mathrm{~nm}$ and excited the sample at normal incidence from the Pt side. The probe beam was frequency doubled to $400 \mathrm{~nm}$ using a beta-barium borate (BBO) crystal and incident with a small angle of $6^{\circ}$ onto the GGG substrate. Both beams were linearly polarized and focused onto the sample in spot diameters of $\sim 260 \mu \mathrm{m}$ for the pump and $\sim 60 \mu \mathrm{m}$ for the probe. The probe wavelength was well above the optical absorption edge of the GGG, which allowed the probe pulses to penetrate the substrate and reach the Bi-YIG layer. After interacting with the Bi-YIG, the reflected probe pulses allowed measuring the differential changes of the magneto-optical polar Kerr rotation $\Delta \Theta_{K}(t)$ and reflectivity $\Delta R(t)$ induced by the excitation pulse as a function of the time delay $t$ between the pump and probe pulses using a lock-in detection scheme. The external magnetic field $H_{\text {ext }}$ was applied perpendicular to the plane of the film. All measurements were performed at room temperature.

\section{RESULTS AND DISCCUSSION}

Figure 3(a) shows the TR-MOKE induced by an incident pump energy density of $E_{\text {pump }}=9.4 \mathrm{mJcm}^{-2}$ for $H_{\text {ext }}=$ $164 \mathrm{mT}$. Three important features can be clearly distinguished. First, the $\Delta \Theta_{\mathrm{K}}$ signal shows a small ultrafast decrease within the first picosecond after the arrival of hot electrons at the $\mathrm{Cu} / \mathrm{Bi}$-YIG interface, followed by a recovery toward the initial state in the next few picoseconds, as 

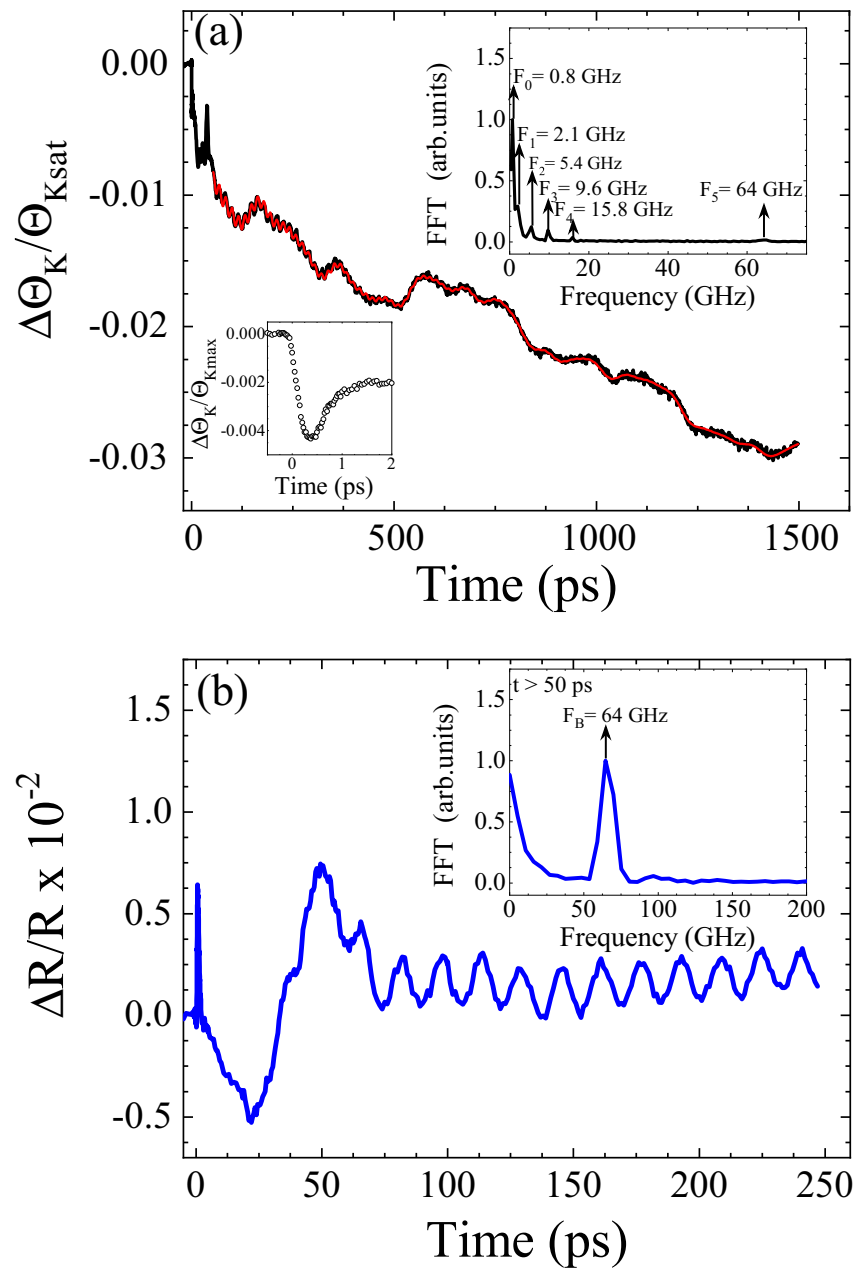

FIG. 3. Dynamics of spin and reflectivity in the $\mathrm{Bi}_{1} \mathrm{Y}_{2} \mathrm{Fe}_{5} \mathrm{O}_{12} / \mathrm{GGG}(100)$ buried below thick $\mathrm{Pt} / \mathrm{Cu}$ bilayers. $\Delta \Theta_{\mathrm{K}} / \Theta_{\mathrm{K}}$ and $\Delta R / R$ induced by a pump energy density of $E_{\text {pump }}=9.4 \mathrm{mJcm}^{-2}$ for $H_{\text {ext }}=164 \mathrm{mT}$. The solid red line in (a) is the fit using Eq. (1). Insets: (a) Fourier transform spectrum of the $\Delta \Theta_{\mathrm{K}} / \Theta_{\mathrm{K}}$ data for the time delay $t \geqslant 50 \mathrm{ps}$ (top) and a magnified view of the $\Delta \Theta_{K} / \Theta_{K}$ signal (bottom) around the zero time delay. (b) Fourier transform spectrum of the $\Delta R / R$ data for the time delay $t \geqslant 50 \mathrm{ps}$ (top).

highlighted in the bottom inset of Fig. 3(a). Second, a gradual slow decrease of the $\Delta \Theta_{\mathrm{K}}$ signal started at $\sim 5$ ps and reached a large value at $1500 \mathrm{ps}$. Third, very complex oscillations are superimposed on the slow exponential change of the $\Delta \Theta_{\mathrm{K}}$ signal. The characteristic times of the fast and slow decrease of the TR-MOKE are $\tau_{\text {fast }}=0.4 \pm 0.09 \mathrm{ps}$ and $\tau_{\text {slow }}=1220 \pm$ $60 \mathrm{ps}$. On the other hand, the complex oscillations are formed by six modes with different amplitudes in the frequency range between 0.8 and $64 \mathrm{GHz}$, as seen in the fast Fourier transform (FFT) spectrum in the top inset of Fig. 3(a). To study these modes in more detail, the TR-MOKE measurements are fitted with the following time damped oscillators:

$$
\begin{aligned}
\Delta \Theta_{\mathrm{K}}(t>41 \mathrm{ps})= & \sum_{i=0}^{5} A_{i} e^{-t / \tau_{i}} \sin \left(2 \pi f_{i} t-\phi_{i}\right) \\
& +B e^{-t / \tau_{\text {slow }}}+C
\end{aligned}
$$

Where $A_{i}, f_{i}, \phi_{i}$, and $\tau_{i}$ are, respectively, the amplitude, frequency, initial phase, and decay time characterizing the oscillation of the mode $i(i=0,1,2,3,4$, and 5). The term $B e^{-t / \tau_{\text {slow }}}$ represents the slow exponential change with the characteristic time $\tau_{\text {slow }}$ and $C$ is an offset. The corresponding fitting with Eq. (1) is plotted in Fig. 3(a) with a red solid line, showing a good agreement with the experimental data. It yields the frequencies $f_{0}=0.8 \mathrm{GHz}, f_{1}=$ $2.2 \mathrm{GHz}, f_{2}=5.4 \mathrm{GHz}, f_{3}=9.6 \mathrm{GHz}, f_{4}=15.5 \mathrm{GHz}$, and $f_{5}=64 \mathrm{GHz}$, which are similar to the ones obtained by the FFT analysis.

To have more information about these modes, we measured the pump-induced change in the reflectivity signal [see Fig. 3(b)]. Clear oscillations with a frequency of $\sim 64 \mathrm{GHz}$ are observed from the time delay $t=41 \mathrm{ps,}$, as seen in the FFT spectrum displayed in the inset of Fig. 3(b). These oscillations can be related to the time-domain Brillouin scattering, which originates from the interference of the probe beam reflected from the Bi-YIG surface with the reflection from the longitudinal strain pulse propagating into the GGG. Indeed, the observed frequency perfectly matches the value $f_{\mathrm{GGG}}=2 V_{\mathrm{GGG}}^{L} \sqrt{n^{2}-\sin ^{2} \theta} / \lambda=63.9 \mathrm{GHz}$, calculated from the probe wavelength $\lambda=400 \mathrm{~nm}$, the longitudinal sound velocity $V_{\mathrm{GGG}}^{L}=6400 \mathrm{~m} / \mathrm{s}$ of GGG [54], its refractive in$\operatorname{dex} n \approx 2[55]$, and the angle of incidence $\theta=6^{\circ}$. Based on these observations, we can conclude that the highest frequency mode in the TR-MOKE is due to the propagation of the longitudinal acoustic pulse in the GGG substrate, since it has the same frequency as the Brillouin oscillation. We note that the observation of Brillouin oscillations in a TR-MOKE signal was previously reported in the literature [29-31,56]. It can be understood considering a small difference in the reflection of right $\left(\sigma^{+}\right)$and left $\left(\sigma^{-}\right)$helicity of light induced by the acoustic pulse during its propagation in the sample [30,56].

The frequencies of the low-frequency modes are in the range of SW frequencies in Bi-YIG films [43,44,57,58]. To clarify the nature of these modes, we investigated their frequencies as a function of the mode number. Interestingly, the precession frequencies of these modes have a quadratic dependence on the mode number. Such a variation is well described by the dispersion relation of SSW [36,37], which is written in the case of our experimental configuration as $[36,37]$ :

$$
\omega(k)=\omega_{0}+\gamma D_{\mathrm{ex}} k^{2}
$$

where $\omega$ is the angular precession frequency, $\omega_{0}$ is the angular frequency of the fundamental mode which depends on $H_{\text {ext }}$ and the effective magnetic anisotropy field, $\gamma$ is the gyromagnetic ratio, $D_{\text {ex }}$ is the exchange stiffness, and $k=n \pi / d$ represents the wave-vector of the standing SWs given by $d=135 \mathrm{~nm}$. Indeed, a fit of experimental data with Eq. (2) using $(\gamma / 2 \pi)=28 \mathrm{GHz} / \mathrm{T}$ yields an excellent agreement with the experimental results (Fig. 4). We obtained an exchange stiffness $D_{\mathrm{ex}}=(5.9 \pm 0.2) 10^{-17} \mathrm{~T} \mathrm{~m}^{2}$ in good agreement with those reported in the literature for YIG and Bi-YIG $[49,59,60]$. This clearly demonstrates the SSW nature of the observed magnetic resonance modes. It also shows that we can excite SSWs with a frequency up to $15 \mathrm{GHz}$, which is 


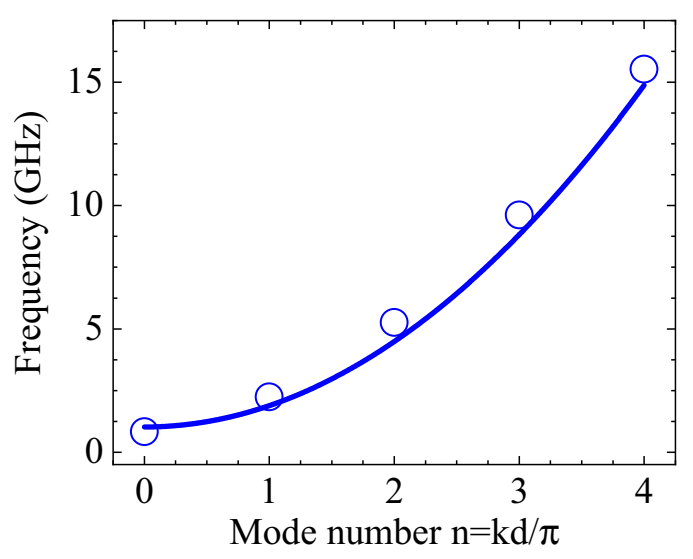

FIG. 4. Spin precession frequency as a function of the mode number. The solid line is the fit obtained using Eq. (2).

15 times higher than the one associated with the ferromagnetic resonance mode (i.e., $n=0$ ). The general excitation mechanism of the SSWs can be related to an ultrafast change of magnetic anisotropy via coherent and incoherent phonons. The coherent phonons correspond to the strain wave shown in Fig. 1(c), and it changes the magnetic anisotropy via the inverse magnetostriction. The incoherent phonons correspond to the phonon-temperature change shown in Fig. 1(d). They change the magnetic anisotropy due to the temperature dependence of magnetic anisotropy constants. We discuss below that the physics behind the generation of incoherent phonons should combine the heat energy directly deposited by the hot-electron pulse at the Bi-YIG surface together with the heat energy transferred from $\mathrm{Cu}$ to $\mathrm{Bi}-\mathrm{YIG}$.

Let us now discuss the fast and slow decrease in the $\Delta \Theta_{\mathrm{K}}$ signal. To give a comprehensive discussion of possible mechanisms behind these phenomena, it is worth mentioning first that the fast decrease and recovery in the $\Delta \Theta_{K}$ signal are similar to the ultrafast demagnetization process usually induced in metallic magnetic films by ultrashort laser [1] and hot-electron [24] excitations. The exact mechanism of this ultrafast demagnetization is still under debate, but different models have been proposed with the most used being the so-called three-temperature model [1]. This model is purely phenomenological and based on the heat transfer between three interacting subsystems representing electrons, lattice (i.e., phonon), and spins. Such an energy transfer leads to an ultrafast increase of spin temperature, which results in an ultrafast decrease of the magnetic order according to the dependence of the magnetization on the temperature. In magnetic insulators where no free electrons are present, the heating of the spin occurs mainly via the phonon-magnon coupling, which is very weak in the case of iron garnets [61-63]. Therefore, this mechanism cannot be the origin of the subpicosecond decrease of the magnetic order. However, the weak phonon-magnon coupling characterizing iron garnets is in good agreement with the slow decrease in the $\Delta \Theta_{\mathrm{K}}$ signal, which can therefore be related to a slow demagnetization process. These slow demagnetization dynamics are consistent with the generation and propagation of incoherent phonons (heat) into the Bi-YIG layer [see Fig. 1(d)]. We note that Fig. 1(d) does not include the process of generating incoherent phonons directly deposited by the hot-electron pulse at the Bi-YIG surface. Including this process in the simulations is a complex task and will essentially increase the temperature in the first nanometer of Bi-YIG, which could compensate a reduced interface conductance for acoustic phonons at the metal-insulator contact. Due to the absence of free electrons in insulators, heat transport in Bi-YIG is identical to incoherent phonon transport. The decrease of the magnetic order is expected to be inhomogeneous across the Bi-YIG thickness and follow the phonon temperature gradient [Fig. 1(d)] with much larger demagnetization close to the $\mathrm{Cu}$ interface.

Apart from the phenomenological three-temperature model, the ultrafast demagnetization dynamics are explained from a microscopic point of view using processes involving fundamental interactions at the origin of magnetism: the spinorbit interaction and the exchange interaction [64-67]. The spin-orbit interaction should only make a small contribution to the ultrafast loss of the magnetic order observed in our sample. Similar ultrafast decrease in the $\Delta \Theta_{K} / \Theta_{K \max }$ signal, i.e., amplitude and timescale, have been measured in heterostructures based on a $\mathrm{Bi}_{1} \mathrm{Y}_{2} \mathrm{Fe}_{5} \mathrm{O}_{12}$ or a $\mathrm{Bi}_{3} \mathrm{Fe}_{5} \mathrm{O}_{12}$ (not shown), in which different spin-orbit couplings are present due to various $\mathrm{Bi}$ concentrations. The large difference in spin-orbit coupling has been experimentally verified in $\mathrm{Bi}_{x} \mathrm{Y}_{3-x} \mathrm{Fe}_{5} \mathrm{O}_{12}$, in which a huge increase of the MO properties was reported when increasing the $\mathrm{Bi}$ concentration $[68,69]$. We therefore suggest that the ultrafast change of the magnetic order is induced by an ultrafast modulation of the exchange interaction [67]. This mechanism has been recently suggested to explain subpicosecond magnetization dynamics induced in YIG and Bi/Ga-substituted YIG by resonant lattice excitation with an ultrashort terahertz pulse and theoretically supported via atomistic spin-dynamics simulations [67]. The scenario behind the modulation of the exchange interaction can be illustrated as follows. The excited incoherent phonons modulate the distance of adjacent atoms, in particular oxygen atoms. Since the superexchange interaction mediated by these atoms is distance dependent, this perturbation leads to an ultrafast modulation of the superexchange interaction between the two iron sublattices, which result in subpicosecond demagnetization dynamics [67].

To investigate further the ultrafast magnetization dynamics, we studied the fast and slow decrease of the magnetic order as a function of the pump energy density and the external magnetic field (see Fig. 5). The amplitude of both processes linearly increases with increasing $E_{\text {pump. }}$. This is in good agreement with the expected dependence of the heat energy density of hot electrons on $E_{\text {pump }}$, since it is the main origin of the heat energy generated in our structure. The small nonlinearity in the heat transport originating from the temperature-dependent electronic heat transport is not relevant here, as confirmed by our UXRD studies [32]. On the other hand, we show that the magnetic field dependence of these amplitudes is the same as the static magnetization of the sample [see Fig. 2(a)]. Indeed, they saturate at $H_{\mathrm{ext}} \sim 260 \mathrm{mT}$, which is the magnetization saturation field characterizing the Bi-YIG film. These results clearly confirm our prediction about the magnetic origin of these phenomena. It is worth mentioning here that the amplitude of the fast demagnetization is about one order of magnitude smaller than the slow 

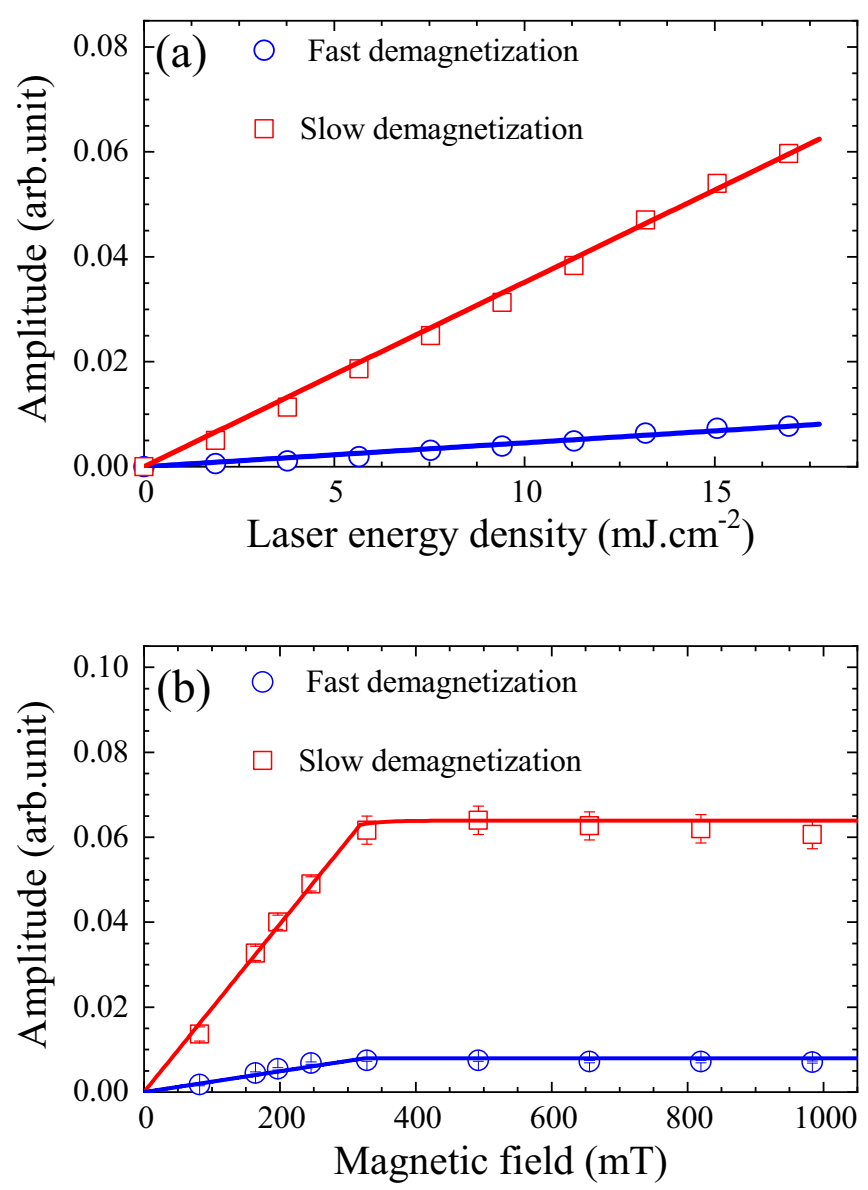

FIG. 5. Amplitude of the fast (circle) and slow (square) demagnetization processes as a function of (a) the pump energy density and (b) the external magnetic field. The solid lines are guides to the eye. demagnetization for all measured values of $H_{\text {ext }}$ and $E_{\text {pump }}$. This is consistent with a very small amplitude of the motion of oxygen atoms, which is in good agreement with the high stiffness and stability characterizing the garnet structure [70,71].

\section{CONCLUSIONS}

We have studied the magnetic, optical, and lattice responses of a $\mathrm{Pt} / \mathrm{Cu} / \mathrm{Bi}_{1} \mathrm{Y}_{2} \mathrm{Fe}_{5} \mathrm{O}_{12} / \mathrm{Gd}_{3} \mathrm{Ga}_{5} \mathrm{O}_{12}$ heterostructure upon femtosecond laser excitation of the opaque $\mathrm{Pt} / \mathrm{Cu}$ bilayers. We find that coherent and incoherent phonons generated by the laser-excited metal electrons trigger highfrequency $\mathrm{SSWs}$ in the $\mathrm{Bi}_{1} \mathrm{Y}_{2} \mathrm{Fe}_{5} \mathrm{O}_{12}$ layer via phononinduced change of the magnetic anisotropy. The incoherent phonons can induce a fast $(<1 \mathrm{ps})$ and slow $(>1000 \mathrm{ps})$ decrease of the magnetic order. We explain these two timescales by considering scenarios based on spin-phonon interaction. Our findings highlight the possibility of triggering highfrequency SWs in magnetic garnets embedded in complex heterostructure devices.

\section{ACKNOWLEDGMENTS}

This paper was supported by the French Investments for the Future Program (PIA) project "Lorraine Université d'Excellence", reference ANR-15-IDEX-04-LUE, the Bundesministerium für Bildung und Forschung Project Grant No. 05K16IPA, and the Deutsche Forschungsgemeinschaft Grant No. BA 2281/11-1. M.D. acknowledges the Alexander von Humboldt Foundation for financial support.
[1] E. Beaurepaire, J. C. Merle, A. Daunois, and J. Y. Bigot, Ultrafast Spin Dynamics in Ferromagnetic Nickel, Phys. Rev. Lett. 76, 4250 (1996).

[2] U. Bovensiepen, Femtomagnetism: magnetism in step with light, Nat. Phys. 5, 461 (2009).

[3] G. Zhang, W. Hübner, E. Beaurepaire, and J.-Y. Bigot, in Spin Dynamics in Confined Magnetic Structures I, edited by B. Hillebrands and K. Ounadjela (Springer Berlin Heidelberg, Berlin, Heidelberg, 2002).

[4] C. D. Stanciu, F. Hansteen, A. V. Kimel, A. Kirilyuk, A. Tsukamoto, A. Itoh, and T. Rasing, All-Optical Magnetic Recording with Circularly Polarized Light, Phys. Rev. Lett. 99, 047601 (2007).

[5] S. Mangin, M. Gottwald, C. H. Lambert, D. Steil, V. Uhlîr, L. Pang, M. Hehn, S. Alebrand, M. Cinchetti, G. Malinowski, Y. Fainman, M. Aeschlimann, and E. E. Fullerton, Engineered materials for all-optical helicity-dependent magnetic switching, Nat. Mater. 13, 286 (2014).

[6] E. Beaurepaire, M. Maret, V. Halté, J. C. Merle, A. Daunois, and J. Y. Bigot, Spin dynamics in $\mathrm{CoPt}_{3}$ alloy films: A magnetic phase transition in the femtosecond time scale, Phys. Rev. B 58, 12134 (1998).
[7] G. Ju, J. Hohlfeld, B. Bergman, R. J. M. van de Veerdonk, O. N. Mryasov, J.-Y. Kim, X. Wu, D. Weller, and B. Koopmans, Ultrafast Generation of Ferromagnetic Order via a Laser-Induced Phase Transformation in FeRh Thin Films, Phys. Rev. Lett. 93, 197403 (2004).

[8] J. A. de Jong, I. Razdolski, A. M. Kalashnikova, R. V. Pisarev, A. M. Balbashov, A. Kirilyuk, T. Rasing, and A. V. Kimel, Coherent Control of the Route of an Ultrafast Magnetic Phase Transition via Low-Amplitude Spin Precession, Phys. Rev. Lett. 108, 157601 (2012).

[9] M. van Kampen, C. Jozsa, J. T. Kohlhepp, P. LeClair, L. Lagae, W. J. M. de Jonge, and B. Koopmans, All-Optical Probe of Coherent Spin Waves, Phys. Rev. Lett. 88, 227201 (2002).

[10] A. V. Kimel, A. Kirilyuk, P. A. Usachev, R. V. Pisarev, A. M. Balbashov, and T. Rasing, Ultrafast non-thermal control of magnetization by instantaneous photomagnetic pulses, Nature 435, 655 (2005).

[11] K. Vahaplar, A. M. Kalashnikova, A. V. Kimel, D. Hinzke, U. Nowak, R. Chantrell, A. Tsukamoto, A. Itoh, A. Kirilyuk, and T. Rasing, Ultrafast Path for Optical Magnetization Reversal via a Strongly Nonequilibrium State, Phys. Rev. Lett. 103, 117201 (2009). 
[12] T. A. Ostler et al., Ultrafast heating as a sufficient stimulus for magnetization reversal in a ferrimagnet, Nat. Commun. 3, 666 (2012).

[13] P. Němec, V. Novák, N. Tesařová, E. Rozkotová, H. Reichlová, D. Butkovičová, F. Trojánek, K. Olejník, P. Malý, R. P. Campion, B. L. Gallagher, J. Sinova, and T. Jungwirth, The essential role of carefully optimized synthesis for elucidating intrinsic material properties of (Ga,Mn)As, Nat. Commun. 4, 1422 (2013).

[14] R. R. Subkhangulov, H. Munekata, T. Rasing, and A. V. Kimel, Laser-induced spin dynamics in ferromagnetic (In,Mn)As at magnetic fields up to 7 T, Phys. Rev. B 89, 060402(R) (2014).

[15] S. Shihab, H. Riahi, L. Thevenard, H. J. V. Bardeleben, A. Lemaitre, and C. Gourdon, Systematic study of the spin stiffness dependence on phosphorus alloying in the ferromagnetic semiconductor (Ga,Mn)As, Appl. Phys. Lett. 106, 142408 (2015).

[16] S. Shihab, L. Thevenard, A. Lemaitre, and C. Gourdon, Counter-rotating standing spin waves: a magneto-optical illusion, Phys. Rev. B 95, 144411 (2017).

[17] F. Hansteen, A. Kimel, A. Kirilyuk, and T. Rasing, Femtosecond Photomagnetic Switching of Spins in Ferrimagnetic Garnet Films, Phys. Rev. Lett. 95, 047402 (2005).

[18] M. Deb, P. Molho, B. Barbara, and J.-Y. Bigot, Temperature and magnetic field dependence of rare-earth $\leftrightarrow$ iron exchange resonance mode in a magnetic oxide studied with femtosecond magneto-optical kerr effect, Phys. Rev. B 94, 054422 (2016).

[19] A. M. Kalashnikova, A. V. Kimel, R. V. Pisarev, V. N. Gridnev, A. Kirilyuk, and T. Rasing, Impulsive Generation of Coherent Magnons by Linearly Polarized Light in the EasyPlane Antiferromagnet $\mathrm{FeBO}_{3}$, Phys. Rev. Lett. 99, 167205 (2007).

[20] F. Hansteen, A. Kimel, A. Kirilyuk, and T. Rasing, Nonthermal ultrafast optical control of the magnetization in garnet films, Phys. Rev. B 73, 014421 (2006).

[21] M. Deb, M. Vomir, J.-L. Rehspringer, and J.-Y. Bigot, Ultrafast optical control of magnetization dynamics in polycrystalline bismuth doped iron garnet thin films, Appl. Phys. Lett. 107, 252404 (2015).

[22] A. B. Chizhik, I. I. Davidenko, A. Maziewski, and A. Stupakiewicz, High-temperature photomagnetism in Codoped yttrium iron garnet films, Phys. Rev. B 57, 14366 (1998)

[23] A. Stupakiewicz, K. Szerenos, D. Afanasiev, A. Kirilyuk, and A. V. Kimel, Ultrafast nonthermal photo-magnetic recording in a transparent medium, Nature 542, 71 (2017).

[24] N. Bergeard, M. Hehn, S. Mangin, G. Lengaigne, F. Montaigne, M. L. M. Lalieu, B. Koopmans, and G. Malinowski, HotElectron-Induced Ultrafast Demagnetization in Co/Pt Multilayers, Phys. Rev. Lett. 117, 147203 (2016).

[25] Y. Xu, M. Deb, G. Malinowski, M. Hehn, W. Zhao, and $\mathrm{S}$. Mangin, Ultrafast magnetization manipulation using single femtosecond light and hot-electron pulses, Adv. Mater. 29, 1703474 (2017).

[26] R. B. Wilson, J. Gorchon, Y. Yang, C.-H. Lambert, S. Salahuddin, and J. Bokor, Ultrafast magnetic switching of GdFeCo with electronic heat currents, Phys. Rev. B 95, 180409(R) (2017).

[27] J.-W. Kim, M. Vomir, and J.-Y. Bigot, Ultrafast Magnetoacoustics in Nickel Films, Phys. Rev. Lett. 109, 166601 (2012).
[28] J.-W. Kim, M. Vomir, and J.-Y. Bigot, Controlling the spins angular momentum in ferromagnets with sequences of picosecond acoustic pulses, Sci. Rep.-Uk 5, 8511 (2015).

[29] T. L. Linnik, V. N. Kats, J. Jäger, A. S. Salasyuk, D. R. Yakovlev, A. W. Rushforth, A. V. Akimov, A. M. Kalashnikova, M. Bayer, and A. V. Scherbakov, The effect of dynamical compressive and shear strain on magnetic anisotropy in a low symmetry ferromagnetic film, Phys. Scripta 92, 054006 (2017).

[30] A. V. Scherbakov, A. S. Salasyuk, A. V. Akimov, X. Liu, M. Bombeck, C. Brüggemann, D. R. Yakovlev, V. F. Sapega, J. K. Furdyna, and M. Bayer, Coherent Magnetization Precession in Ferromagnetic (Ga,Mn)As Induced by Picosecond Acoustic Pulses, Phys. Rev. Lett. 105, 117204 (2010).

[31] M. Deb, E. Popova, M. Hehn, N. Keller, S. Mangin, and G. Malinowski, Picosecond acoustic-excitation-driven ultrafast magnetization dynamics in dielectric Bi-substituted yttrium iron garnet, Phys. Rev. B 98, 174407 (2018).

[32] J.-E. Pudell, M. Mattern, M. Hehn, G. Malinowski, M. Herzog, and M. Bargheer, Heat transport without heating?-An ultrafast $\mathrm{X}$-ray perspective into a metal heterostructure, Adv. Funct. Mater. 30, 2004555 (2020).

[33] I. L. Markov, Limits on fundamental limits to computation, Nature 512, 147 (2014).

[34] A. G. Gurevich and G. A. Melkov, Magnetization Oscillations and Waves (CRC Press, Boca Raton, 1996).

[35] A. A. Serga, A. V. Chumak, and B. Hillebrands, YIG magnonics, J. Phys. D: Appl. Phys. 43, 264002 (2010).

[36] C. Kittel, Introduction to Solid State Physics (John Wiley \& Sons, New York, 1986).

[37] A. H. Morrish, The Physical Principles of Magnetism (IEEE, New York, 2001).

[38] I. S. Maksymov and M. Kostylev, Broadband stripline ferromagnetic resonance spectroscopy of ferromagnetic films, multilayers and nanostructures, Physica E 69, 253 (2015).

[39] Y. Ding, T. J. Klemmer, and T. M. Crawford, A coplanar waveguide permeameter for studying high-frequency properties of soft magnetic materials, J. Appl. Phys. 96, 2969 (2004).

[40] H. Yu, O. d' Allivy Kelly, V. Cros, R. Bernard, P. Bortolotti, A. Anane, F. Brandl, F. Heimbach, and D. Grundler, Approaching soft X-ray wavelengths in nanomagnet-based microwave technology, Nat. Commun. 7, 11255 (2016).

[41] J. Walowski, M. D. Kaufmann, B. Lenk, C. Hamann, J. McCord, and M. Münzenberg, Intrinsic and non-local Gilbert damping in polycrystalline nickel studied by $\mathrm{Ti}$ : sapphire laser fs spectroscopy, J. Phys. D: Appl. Phys. 41, 164016 (2008).

[42] B. Lenk, G. Eilers, J. Hamrle, and M. Münzenberg, Spin-wave population in nickel after femtosecond laser pulse excitation, Phys. Rev. B 82, 134443 (2010).

[43] M. Deb, E. Popova, M. Hehn, N. Keller, S. Petit-Watelot, M. Bargheer, S. Mangin, and G. Malinowski, Femtosecond Laser-Excitation-Driven High Frequency Standing Spin Waves in Nanoscale Dielectric Thin Films of Iron Garnets, Phys. Rev. Lett. 123, 027202 (2019).

[44] M. Deb, E. Popova, M. Hehn, N. Keller, S. Petit-Watelot, M. Bargheer, S. Mangin, and G. Malinowski, Damping of Standing Spin Waves in Bismuth-Substituted Yttrium Iron Garnet as Seen via the Time-Resolved Magneto-Optical Kerr Effect, Phys. Rev. Appl. 12, 044006 (2019).

[45] E. Popova, L. Magdenko, H. Niedoba, M. Deb, B. Dagens, B. Berini, M. Vanwolleghem, C. Vilar, F. Gendron, A. Fouchet, 
J. Scola, Y. Dumont, M. Guyot, and N. Keller, Magnetic properties of the magnetophotonic crystal based on bismuth iron garnet, J. Appl. Phys. 112, 093910 (2012).

[46] M. Bauer, A. Marienfeld, and M. Aeschlimann, Hot electron lifetimes in metals probed by time-resolved two-photon photoemission, Prog. Surf. Sci. 90, 319 (2015).

[47] D. Schick, A. Bojahr, M. Herzog, C. V. K. Schmising, R. Shayduk, W. Leitenberger, P. Gaal, and M. Bargheer, Normalization schemes for ultrafast $\mathrm{x}$-ray diffraction using a table-top laser-driven plasma source, Rev. Sci. Instrum. 83, 025104 (2012).

[48] D. Schick, A. Bojahr, M. Herzog, R. Shayduk, C. von Korff Schmising, and M. Bargheer, UDKM1DsIM-A simulation toolkit for 1D ultrafast dynamics in condensed matter, Comput. Phys. Commun. 185, 651 (2014).

[49] G. G. Siu, C. M. Lee, and Y. Liu, Magnons and acoustic phonons in $\mathrm{Y}_{3-x} \mathrm{Bi}_{x} \mathrm{Fe}_{5} \mathrm{O}_{12}$, Phys. Rev. B 64, 094421 (2001).

[50] S. P. Zeuschner, J. E. Pudell, A. von Reppert, M. Deb, E. Popova, N. Keller, M. Rössle, M. Herzog, and M. Bargheer, Measurement of transient strain induced by two-photon excitation, Phys. Rev. Res. 2, 022013 (2020).

[51] M. Schreier, A. Kamra, M. Weiler, J. Xiao, G. E. W. Bauer, R. Gross, and S. T. B. Goennenwein, Magnon, phonon, and electron temperature profiles and the spin seebeck effect in magnetic insulator/normal metal hybrid structures, Phys. Rev. B 88, 094410 (2013).

[52] S. A. Manuilov, S. I. Khartsev, and A. M. Grishin, Pulsed laser deposited $\mathrm{Y}_{3} \mathrm{Fe}_{5} \mathrm{O}_{12}$ films: nature of magnetic anisotropy I, J. Appl. Phys. 106, 123917 (2009).

[53] S. A. Manuilov and A. M. Grishin, Pulsed laser deposited $\mathrm{Y}_{3} \mathrm{Fe}_{5} \mathrm{O}_{12}$ films: nature of magnetic anisotropy II, J. Appl. Phys. 108, 013902 (2010).

[54] V. F. Kitaeva, E. V. Zharikov, and I. L. Chistyi, The properties of crystals with garnet structure, Phys. Status Solidi A 92, 475 (1985).

[55] D. L. Wood and K. Nassau, Optical properties of gadolinium gallium garnet, Appl. Optics 29, 3704 (1990).

[56] L. Thevenard, E. Peronne, C. Gourdon, C. Testelin, M. Cubukcu, E. Charron, S. Vincent, A. Lemaître, and B. Perrin, Effect of picosecond strain pulses on thin layers of the ferromagnetic semiconductor $(\mathrm{Ga}, \mathrm{Mn})(\mathrm{As}, \mathrm{P})$, Phys. Rev. B 82, 104422 (2010).

[57] S. Klingler, V. Amin, S. Geprägs, K. Ganzhorn, H. Maier-Flaig, M. Althammer, H. Huebl, R. Gross, R. D. McMichael, M. D. Stiles, S. T. B. Goennenwein, and M. Weiler, Spin-Torque
Excitation of Perpendicular Standing Spin Waves in Coupled YIG/Co Heterostructures, Phys. Rev. Lett. 120, 127201 (2018).

[58] H. Qin, S. J. Hämäläinen, and S. van Dijken, Exchange-torqueinduced excitation of perpendicular standing spin waves in nanometer-thick YIG films, Sci. Rep.-Uk 8, 5755 (2018).

[59] A. Gurevich and A. Anisimov, Intrinsic spin wave relaxation processes in yttrium iron garnets, Sov. Phys. JETP 41, 336 (1975).

[60] S. Klingler, A. V. Chumak, T. Mewes, B. Khodadadi, C. Mewes, C. Dubs, O. Surzhenko, B. Hillebrands, and A. Conca, Measurements of the exchange stiffness of YIG films using broadband ferromagnetic resonance techniques, J. Phys. D Appl. Phys. 48, 015001 (2014).

[61] E. G. Spencer and R. C. LeCraw, Spin-Lattice Relaxation in Yttrium Iron Garnet, Phys. Rev. Lett. 4, 130 (1960).

[62] D. L. Huber, Spin-magnon relaxation in rare-earth iron garnets, Phys. Rev. 136, A500 (1964).

[63] G. F. Dionne and G. L. Fitch, Temperature dependence of spinlattice relaxation in rare-earth iron garnets, J. Appl. Phys. 87, 4963 (2000).

[64] B. Koopmans, G. Malinowski, F. Dalla Longa, D. Steiauf, M. Fahnle, T. Roth, M. Cinchetti, and M. Aeschlimann, Explaining the paradoxical diversity of ultrafast laser-induced demagnetization, Nat. Mater. 9, 259 (2010).

[65] K. Krieger, J. K. Dewhurst, P. Elliott, S. Sharma, and E. K. U. Gross, Laser-induced demagnetization at ultrashort time scales: predictions of TDDFT, J. Chem. Theory Comput. 11, 4870 (2015).

[66] G. P. Zhang, M. S. Si, Y. H. Bai, and T. F. George, Magnetic spin moment reduction in photoexcited ferromagnets through exchange interaction quenching: beyond the rigid band approximation, J. Phys. Condens. Matter 27, 206003 (2015).

[67] S. F. Maehrlein, I. Radu, P. Maldonado, A. Paarmann, M. Gensch, A. M. Kalashnikova, R. V. Pisarev, M. Wolf, P. M. Oppeneer, J. Barker, and T. Kampfrath, Dissecting spin-phonon equilibration in ferrimagnetic insulators by ultrafast lattice excitation, Sci. Adv. 4, eaar5164 (2018).

[68] P. Hansen and J. P. Krumme, Magnetic and magneto-optical properties of garnet films, Thin Solid Films 114, 69 (1984).

[69] S. Wittekoek, T. J. A. Popma, J. M. Robertson, and P. F. Bongers, Magneto-optic spectra and the dielectric tensor elements of bismuth-substituted iron garnets at photon energies between 2.2-5.2 eV, Phys. Rev. B 12, 2777 (1975).

[70] G. D. Winkler, Magnetic Garnets, (Vieweg, Braunschweig, 1981).

[71] G. F. Dionne, Magnetic Oxides, (Springer, New York, 2009). 